CENTENARIO

\title{
Palabras en el Centenario
}

\section{Speech delivered at the 100th anniversary of Havana's Dental Surgery School}

\section{Luis Suárez Rosas}

Decano, Facultad de Estomatología de la Habana. La Habana, Cuba.

\author{
Ministro \\ Rectores y Decanos \\ Distinguidos Profesores \\ Queridos alumnos \\ Estimados invitados \\ Compañeras y Compañeros todos
}

Ha querido la vida y las circunstancias que sobre los hombros de este humilde servidor recayera la enorme responsabilidad de pronunciar las palabras que recordaran en esta sesión conmemorativa, hoy domingo 19 de Noviembre del 2000, el hecho trascendental para la Docencia Universitaria de Cuba y del Mundo que significó la fundación de la Escuela de Cirugía Dental de la Universidad de La Habana.

Fue precisamente la hermosa Ciudad de La Habana, la Ciudad de las Columnas, donde lo real y lo maravilloso, como señalara el insigne escritor cubano Alejo Carpentier, se entretejen para dar un toque de cubanía sin igual, inolvidable para todos aquellos que la conocen y consecuentemente la aman, la ciudad donde comenzó esta historia que hoy celebramos todos aquellos que tenemos la dicha de compartir este inolvidable momento.

Efectivamente, el 19 de Noviembre del año 1900 se consumaban toda una serie de aspiraciones de nuestros abnegados y preclaros precursores al fundarse la Escuela de Cirugía Dental anexa a la Facultad de Medicina y Farmacia de la Universidad de La Habana en el viejo caserón, sede de esta Facultad, ubicado en la intersección de las calles Zanja y Belascoaín.

La historia de la docencia y la praxis de la Odontología y la Estomatología en Cuba está llena de hechos relevantes entre los que se destaca la fundación en 1728 de la Real y Pontificia Universidad de San Jerónimo de La Habana, alto centro de estudios 
que se constituiría como el Alma Máter de los Centros Universitarios que posteriormente, y sobre todo a partir de 1959 se diseminan por toda nuestra querida Patria.

Se iniciaba entonces un largo camino preñado de luchas y de aspiraciones para cada etapa histórica que pasa por momentos tales como la creación de la carrera agregada de Flebotomiano en 1842 al secularizarse nuestra Universidad, la cual sería entonces denominada Real y Literaria Universidad de La Habana.

Otro momento importante en esta larga cadena de hechos lo es sin lugar a dudas la decisión de establecer los estudios universitarios para alcanzar el título de Ministrante Dentista tomando por base el Real Decreto de 15 de Junio de 1863 que implicaba la aparición de un Plan de Estudio que propiciaría que por vez primera apareciera la denominación de Dentistas en un currículo universitario en Cuba y en todo el Reino de España.

Todo este proceso no culminaría hasta el año de 1880 que es cuando en Cuba se dicta la Real Orden de 24 de Febrero que hace extensivo a nuestra Isla el Real Decreto de 4 de Junio de 1875 por el cual se crea la profesión de Cirujano Dentista en España.

En este necesario recuento existe una fecha y un nombre que los que abrazamos esta humana y solidaria carrera no podemos olvidar. Me refiero al año de 1878 y al Dr. Juan García Villarraza, médico y dentista que inauguró la primera Academia Dental en La Habana amparado por la autorización de la Metrópolis para abrir este tipo de instituciones que enseñaran el arte dentario de la época y preparara a los cursantes a examinarse ante Tribunales Universitarios donde demostraran sus capacidades y aptitudes para el ejercicio de la profesión. El surgimiento y el desarrollo de estas Academias, entre las que se destacan además las de los doctores Florencio Cancio e Ignacio Rojas Quintana conjuntamente con la aparición de diferentes Revistas Científicas y Sociedades del área de nuestra profesión, fortalecieron el prestigio de la Odontología y sirvieron como antecedentes a la fundación de nuestra institución universitaria.

Correspondió a los profesores Pedro Calvo Castellanos, Cirilo Yarini y Ponce de León y a Marcelino Weiss Gramatges el honor y la gloria de ser los fundadores el 19 de Noviembre del 1900 de nuestra histórica Escuela de Cirugía Dental. Con tres unidades dentales, seis alumnos y el decidido apoyo del Secretario de Instrucción y Bellas Artes de la época, el insigne pedagogo cubano Dr. Enrique J osé Varona, además de la colaboración de destacados dentistas entre los que sobresalió el Dr. Federico Poey Aguirre, comenzó esta larga historia que conlleva una tradición de excelencia en la formación de odontólogos y estomatólogos que comienza en el nostálgico y viejo caserón de Zanja y Belascoaín y continua hasta el momento en las instalaciones actuales de la Facultad de Estomatología.

El espíritu de lucha que supieron transmitir los precursores de esta gesta, los fundadores de la Escuela y sus colaboradores, los que posibilitaron junto al Dr. Rafael Biada Dini, primer Decano de nuestro Centro, que en 1937 la institución alcanzara la condición de Facultad de Odontología de la Universidad de La Habana y todos aquellos profesionales, estudiantes y trabajadores que han tenido una participación activa en el surgimiento, desarrollo y consolidación de la Escuela Cubana de Estomatología, se ha constituido como una labor de verdadero apostolado que va más allá de los muros universitarios y se inserta en nuestra sociedad, en nuestro pueblo, en la historia de nuestra Patria. 
No es posible, en el marco limitado de estas palabras, dar una idea exacta de la proyección nacional e internacional que ha alcanzado la Escuela Cubana de Estomatología, que nació sin lugar a dudas de los hechos históricos que reseñamos y que conmemoramos hoy 19 de Noviembre. Es una obra humana que ha dejado una huella que trasciende a los hombres y mujeres que la realizaron y la continúan.

Es una obra que se pone de manifiesto en la vida y la obra del Dr. Oscar Amoedo Valdés, una de las grandes figuras universales de las Ciencias Médicas a la cual el mundo está en deuda constante por su labor científica relacionada fundamentalmente con la Odontología Legal de la cual es su precursor.

El Dr. Amoedo llegó a ser en 1907 Presidente de la Sociedad Odontológica Francesa y ocupando una cátedra titular de la Escuela Dental de París se le planteó el problema de que los Profesores tenían que ser nativos o ciudadanos de Francia. Amoedo respondió que él se negaba a renunciar a su ciudadanía cubana y se decidió entonces a dejar su cargo de Profesor. Este hecho no se consumó porque el Gobierno francés atendiendo al prestigio de nuestro compatriota resolvió el conflicto autorizándole a ejercer su magisterio sin renunciar a su ciudadanía cubana. Fue uno de los activistas mas sobresalientes en el apoyo a la Guerra de Independencia de nuestro pueblo entre los emigrados cubanos de París. Su cubanía siempre lo acompañó. Hoy le rendimos tributo de homenaje y recordación.

Esta obra se pone de manifiesto en la vida y trayectoria de numerosos cubanos que como Federico Poey Aguirre, hijo del sabio naturalista Felipe Poey, desarrollaron una fecunda labor científica dentro y fuera de nuestras fronteras dándole prestigio y relieve a nuestra profesión.

Se detecta en la obra del Dr. Andrés Weber de la Torre que lo hace merecedor de ocupar un escaño en la célebre Academia de Ciencias de Cuba en el año de 1923

Se palpa esta historia en la ejecutoria científica del Dr. Florestán Aguilar, nacido en La Habana en 1872, que fundara la Facultad de Odontología de la Universidad Complutense de Madrid y a quien se recuerda día a día en esa prestigiosa institución universitaria.

Se respira en la vida del Dr. Ismael Clark y Mascaró, primer Profesor de Mérito de nuestra Facultad, que con una interminable hoja de servicios a la Universidad y a la profesión también diseñó el emblema de nuestra Institución conjugando los colores de nuestra enseña nacional y señalando los valores éticos, morales y humanos de la Estomatología.

Es una obra que se refleja en la destacada presencia de los odontólogos y estomatólogos cubanos en todas las epopeyas libertarias de nuestro pueblo, comenzando desde las luchas independentistas del Siglo XIX, donde existieron cuatro dentistas que alcanzaron los grados de General, sobresaliendo entre éstos el Mayor General Emilio Núñez Rodríguez, General de las guerras de 1868, 1878 y 1895, J efe del Departamento de Expediciones del Partido Revolucionario Cubano de J osé Martí, el hombre que más expediciones organizó y trajo a Cuba y cuya destacada trayectoria patriótica le hizo acreedor del honor de izar en el Castillo del Morro la bandera cubana al cese de la ocupación militar norteamericana el 20 de Mayo de 1902 mientras el Generalísimo Máximo Gómez izaba la enseña nacional en el Palacio de los Capitanes Generales.

Se vislumbra esta obra en la corta vida del estudiante del Cuarto año de Odontología Raúl González Sánchez, joven con un profundo amor por su tierra 
cubana y por la libertad asesinado el 2 de Septiembre de 1958 en los momentos en que pretendía incorporarse a las tropas del Directorio Revolucionario 13 de Marzo que luchaban en las montañas del Escambray contra la dictadura batistiana. Su recuerdo y su presencia están para siempre en las clínicas, laboratorios y aulas de la Facultad de Estomatología de La Habana que se honra en llevar su nombre.

La huella de estos pasos de la historia se detecta en la presencia estudiantil de nuestra Facultad en la Directiva de la Federación Estudiantil Universitaria de la Universidad de La Habana en momentos difíciles, convulsos y complejos para nuestro país; en la participación de graduados de nuestras aulas en la gesta del 26 de Julio de 1953; en la clandestinidad y en la lucha guerrillera en las montañas de la Sierra Maestra; en la respuesta de todos aquellos estomatólogos que se negaron a abandonar a su pueblo y a su Universidad y permanecieron en Cuba renunciando a posiciones cómodas y privilegios decidiendo dejar sus huesos en la tierra que los vio nacer abrazados a la estrella que ilumina y mata.

Esta obra de cien años se ve en la colaboración internacional con diferentes países hermanos donde se han constituido Facultades de Estomatología o donde existen Proyectos de colaboración beneficiosos para las instituciones académicas y para los pueblos con los cuales nuestro país colabora.

Esta obra alcanza niveles no imaginados por los precursores y fundadores cuando se analizan los indicadores de salud bucal obtenidos gracias al esfuerzo de profesionales, técnicos y trabajadores en general que laboran en las instituciones estomatológicas del Sistema Nacional de Salud de Cuba.

De manera que estamos hablando de una Huella Histórica de la Estomatología Cubana.

Esta huella ha calado hondo en nuestro pueblo y estoy seguro que también más allá de nuestras fronteras. En su ejecutoria han intervenido cubanos y también personas no nacidas en Cuba. Unos con más profundidad, otros con menos profundidad pero finalmente es una huella.

La Facultad de Estomatología de La Habana, por decisión unánime de su claustro y de su asamblea de estudiantes y trabajadores decidió en fecha tan memorable como la que hoy celebramos instituir la Moneda Conmemorativa "La Huella Histórica de la Estomatología Cubana" y entregarla a personalidades que hayan contribuido con su obra, vida y pensamiento al desarrollo de la Estomatología.

\section{El Maestro J osé Martí proclamó "Honrar Honra."}

Al honrar a los que en el día de hoy reciban la Moneda Conmemorativa "La Huella Histórica de la Estomatología Cubana", como símbolo del reconocimiento por su labor destacada y a los que se les entregue más adelante en actos solemnes en sus respectivos territorios, la Facultad de Estomatología de La Habana "Raúl González Sánchez" se honra.

Compañeras y compañeros todos:

Nunca como antes la Universidad cubana y la Universidad Latinoamericana ha estado tan comprometida con el desarrollo de nuevas prácticas sociales que estén al servicio de la población y de los intereses más nobles, altruistas y solidarios. 
La formación de recursos humanos para las ciencias de la salud dirigida hacia el constante mejoramiento de la salud integral de nuestras poblaciones, requiere hoy en día de un mayor compromiso por parte de todos los que tenemos algún grado de responsabilidad en ello.

La necesidad de integrarnos y cumplir así el sueño de Bolívar y Martí se impone.

Nadie albergue la menor duda de que la Facultad de Estomatología de La Habana en este nuevo siglo que comienza continuará con sus mejores tradiciones históricas. No por gusto han existido hombres y mujeres íntegros en estos cien años que han predicado con el valor de sus vidas como ejemplos y han desbrozado el largo y difícil camino por el cual más de una generación de estomatólogos hemos transitado y por el cual se aprestan a transitar muchos más.

La Estomatología Cubana continuará profundizando su Huella Histórica y se seguirá haciendo camino al andar como señalara el poeta Antonio Machado, enfrentando cualquier tipo de dificultad con entereza y valentía acorde al legado de nuestros precursores.

Los profesores, estudiantes y trabajadores de la Facultad de Estomatología de La Habana al igual que los que pertenecen a las Facultades de Estomatología de Santiago de Cuba, Villa Clara, Camagüey y los Departamentos Docentes de Estomatología de las diferentes Facultades de Ciencias Médicas de Cuba, retoños directos de la obra humana que nació un día como hoy hace cien años al igual que todo el formidable Ejército de la Salud del Sistema Nacional de Salud cubano, continuaremos siendo, como proclamara el Comandante en J efe Fidel Castro Ruz en el acto de graduación de Ciencias Médicas celebrado el 13 de Agosto del presente año:

...abanderados del honor de la Patria, heraldos de la salud y la vida, vencedores de la muerte, campeones olímpicos del verdadero humanismo, que señalan el camino del mundo de mañana, sin explotación, sin saqueo, sin el genocidio de un orden económico y político mundial injusto que mata cada año de enfermedad y hambre a decenas de miles de niños y adultos. 